\title{
Individual heterogeneity in mortality mediates long-term persistence of a seasonal microparasite
}

\author{
Christopher J. Dugaw $\cdot$ Karthik Ram
}

Received: 16 February 2010/ Accepted: 3 November 2010/Published online: 27 November 2010

(C) The Author(s) 2010. This article is published with open access at Springerlink.com

\begin{abstract}
One of the primary objectives in population ecology is to understand mechanisms that allow a species to persist or to be driven to extinction. In most population models, individuals are assumed to be equivalent within any particular category such as age, sex, or morphological grouping. Individuals within such groupings, however, may exhibit considerable variation in traits that can significantly affect population trajectories. Although ecologists have long been aware of such variation, they are frequently ignored to maintain computational tractability. The few statistical models that do incorporate such heterogeneity require prohibitively large amounts of data on many individuals, making them impractical. In California's coastal prairie, a parasitic nematode, Heterorhabditis marelatus, is an important natural enemy, whose presence determines the strength and extent of a trophic cascade. Mortality of
\end{abstract}

Communicated by Roland Brandl.

Electronic supplementary material The online version of this article (doi:10.1007/s00442-010-1844-5) contains supplementary material, which is available to authorized users.

C. J. Dugaw $(\bowtie)$

Department of Mathematics, Humboldt State University,

Arcata, CA 95521, USA

e-mail: dugaw@humboldt.edu

K. Ram

Section of Evolution and Ecology, University of California,

Davis, Davis, CA 95616, USA

K. Ram

Bodega Marine Laboratory, University of California, Davis, Bodega Bay, CA 94923, USA

K. Ram

Department of Environmental Studies, University of California,

Santa Cruz, Santa Cruz, CA 95064, USA
H. marelatus is strongly influenced by habitat and seasonality, which determines long-term persistence. Prior efforts to estimate mortality have suffered from difficulty in distinguishing between measurement and process error due to limitations in experimental protocol. In this study, we eliminate measurement error in the initial population size and focus on the true nature of the heterogeneity in mortality. By including individual heterogeneity in our statistical model, we are able to understand how this species is able to persist over seasonally harsh environmental conditions. Further, we extrapolate these findings to larger population sizes and illustrate that heterogeneous survival can have a significant effect on the emergent number of survivors.

Keywords Heterogeneity - Mortality · Survivorship · Pareto $\cdot$ Weibull $\cdot$ Survival analysis

\section{Introduction}

Biologists often rely on information about births, deaths, and survival rates to predict the abundance and dynamics of a species. One of the primary goals of population ecology is to identify mechanisms that allow species to persist or drive them to extinction. Trends in populations are often determined by fitting count data from observational (Anderson et al. 2001) or experimental studies (Grear and Schmitz 2005) to a set of a priori distributions. Rates derived from such models are used to predict species trajectories and guide conservation efforts (Connors and White 1999).

Within a particular population, individuals typically exhibit phenotypic and genotypic variation between sexes and age-classes (Schoener 1986). Such forms of variation 
have long been incorporated into models of population growth and community dynamics (Czáran 1998). In these models, the assumption is that individuals within a group are ecologically equivalent. Individuals, however, can exhibit variation in the ability to reproduce or survive that extend well beyond the effects of age or sex (Darwin 1859; Lomnicki 1980; Pfister and Stevens 2003). Thus, using models that ignore such variation can lead to incorrect predictions. While estimates of vital rates are measured on individuals, such data are often aggregated into a few parameters as a way to understand overall population dynamics. In such cases, variation among individuals that cannot be attributed to age, sex, or a pre-defined group becomes lost as noise.

Bolnick et al. (2003) highlighted the importance of individual specialization for a range of ecological and evolutionary processes. Heterogeneity among individuals in a population can have important consequences for the extinction risk of a population. Heterogeneous populations are more likely, than homogenous populations, to contain one or more fit individuals with a low probability of mortality. Despite a significant drop in population numbers, such individuals may likely survive and prevent the population from becoming extinct. Thus, ignoring heterogeneity can potentially lead to incorrect predictions about population persistence, stability, or extinction risk (Fox and Kendall 2002). Models that incorporate heterogeneity are therefore important to the conservation and management of small populations (Connors and White 1999). Structured variation, defined as correlation among individuals at one time and correlation within individuals over time, can reduce extinction risk in small populations (Fox et al. 2006). However, the net effect of individual heterogeneity may not be positive depending on other demographic parameters (Vindenes et al. 2008). The magnitude of such effects can be particularly large when environmental heterogeneity is high (Kendall and Fox 2003). In addition to biological heterogeneity, there can be abiotic, environmental heterogeneity. Although experimental ecologists try to minimize variation in experimental designs (Pickett et al. 2000), small-scale heterogeneity is often unavoidable and can affect population processes (Pacala 1987).

We develop a model to estimate mortality of an extinction-prone seasonal microparasite. Heterorhabditis marelatus, an entomopathogenic nematode (EPN), is the most significant natural enemy of a lepidopteran host, Hepialus californicus, in California coastal prairies (Ram et al. 2008a). H. marelatus exist in nature as third instar dauer larvae: non-feeding infective juveniles (IJ). EPN actively move through the soil in search of hosts by relying on a suite of chemical cues (Lewis et al. 2006). IJ do not transition into other physiological states, and reproduction and development can occur only inside a host. A single IJ is capable of producing upwards of 100,000 offspring from a single host (Preisser 2003), although hosts are typically infected by more than one IJ (Ram et al. 2008b). Each IJ must therefore rely on a fixed lipid reserve for survival and movement until it can locate and infect a host (Qiu and Bedding 2000). Under stressful abiotic conditions, infective juveniles exhaust their lipid reserves and rapidly die or fail to successfully infect a host (Elliot 1954). Thus, unfavorable conditions over extended periods can extirpate local populations of IJ. Favorable conditions, on the contrary, allow IJ to conserve lipids and persist over longer periods of time (Qiu and Bedding 2000), thereby increasing their probability of encountering a host (Preisser et al. 2006). Thus, the individuals that endure abiotic stress and survive are more likely to find a host and replenish the local population. Additional natural history about this system can be found in Strong (1999), Preisser et al. (2006), and Ram et al. (2008a, b)

In previous efforts, our research group estimated mortality of IJ by placing an approximate number (via a dilution series) in enclosures and counting the number of survivors over time (Preisser et al. 2006; Ram et al. 2008b). With such a design, we were unable to separate the measurement error of initial population size from process stochasticity. Thus, to obtain an unbiased estimate of the mortality that occurs initially, we incorporated a fixed initial number of $\mathrm{IJ}$ in our experimental design. Initial number of nematodes in experimental tubes did not have any density-dependent effects on survival (Preisser et al. 2005). Since infective juveniles do not have any age or stage structure, we previously assumed that all individuals within an enclosure had identical survival probabilities.

The importance of individual heterogeneity in mortality has been recognized in the study of longevity and senescence (Fox et al. 2006; Service et al. 1998; Vaupel and Carey 1993). The statistical models used in these studies typically assume that an individual's mortality risk varies with age according to some functional form called the hazard function (e.g., Gompertz or Weibull). Each individual's hazard function is multiplied by a constant frailty factor that is assumed to follow a distribution (typically Gamma). These models require data on the lifespan of a very large number of individuals to distinguish between hazard functions. Consequently, these models have limited applicability in most ecological contexts.

Of the few existing models that incorporate individual heterogeneity, few are parameterized by data from survival experiments. Some authors have directly simulated the statistical models to gain greater insight to these survival experiments, but these simulations do not include reproduction (Kowald and Kirkwood 1993; Service et al. 1998). General analytic and simulation models have yielded interesting but differing results that arise from varying 
assumptions about the nature of heterogeneity (Connors and White 1999; Fox 2005; Robert et al. 2003; Vindenes et al. 2008). There is a need to bring the models and experiments together to generate clear conclusions for specific organisms or types of organisms. We argue that this process has been hampered by the complexity of the statistical analysis and the type of data needed.

In this paper, we implement a statistical model of mortality that incorporates heterogeneity in mortality in destructively sampled populations. Incorporating heterogeneity allows for accurate estimates of the extinction probability of the microparasite which consequently determines the nature and strength of this trophic cascade (additional natural history in Preisser 2003; Ram et al. 2008a; Strong 1999; Strong et al. 1996). As emphasized by Duchateau and Janssen (2005), we use hierarchical models that compare heterogeneity in survival at the individual scale, and the sample unit scale with those that do not. We make simplifying assumptions about the nature of heterogeneity sample unit scale, but test our statistical methods against a more mechanistic model using monte carlo simulations. Our modeling approach combines existing statistical models to generate greater insight into the biology of our system.

\section{Materials and methods}

Field methods

We designed our experiment to measure survivorship under the yellow bush lupine (Lupinus arboreus), the favorable habitat for H. marelatus, and under grasslands, the surrounding matrix habitat (Preisser et al. 2005; Ram et al. 2008b). In previous iterations of this experiment, we inoculated experimental tubes with a large number (ca. 1,100) of IJ using a dilution series (Preisser et al. 2005; Ram et al. 2008b). The technique, however, does not yield a precise initial number of individuals, and when combined with the high initial mortality that IJ experience, limited our ability to interpret model results.

As the objective of this experiment was to accurately estimate short-term mortality, we started with a low initial number of 100 IJ. The low number allowed us to accurately count IJ using a precision micropipette (Drummond digital microdisperser, $100 \mu \mathrm{L}$, Fisher Scientific \#21-169-20D). We placed them into sterile soil in a centrifuge tube modified so that the IJ experienced surrounding abiotic conditions without allowing hosts to enter (detailed description in Preisser et al. 2006). Thus, no reproduction occurred over the duration of the experiment. EPN experience high initial mortality from the stress of introduction, both in natural and experimental contexts, but the effects typically last only for a few hours (Smits 1996). We sampled experimental tubes $(n=30$ per treatment) after $2,4,8,16,32$, and 64 days in the field.

To enumerate surviving IJ, we first baited each tube with four Galleria larvae. After allowing the larvae to remain in the tubes for 1 week, we dissected any infected hosts and counted all the IJ inside. We replenished each tube with four fresh larvae and repeated the assay 2 times. Previous experiments demonstrated that three rounds of baiting are adequate to extract $>99 \%$ of surviving IJ (Preisser et al. 2006; Ram et al. 2008b). Our choice of protocol for nematode counting was strongly motivated by the biology of the species. Direct extraction of entomopathogenic nematodes from soil is very inefficient and results in a low recovery rate even under ideal conditions. In laboratory trials, our research group recovered $<50 \%$ of EPN from sterile soil within an hour of inoculation (McLaughlin, unpublished). Kung and Gaugler (1990, 1991) also report comparably low estimates (55-65\%) for related EPN species. While such methods are useful in other contexts, they are inappropriate for quantifying individuals in survivorship studies. Since only a fraction of surviving nematodes can successfully infect and kill a host (Campbell et al. 1999), our bioassay is the only practical way to quantify 'effective' population size at any given time.

Statistical methods

Here, we develop a suite of statistical models to assess three aspects of EPN survival in our experiment: betweentube heterogeneity (i.e. overdispersion), individual heterogeneity (within tube), and effect of rhizosphere type (grassland or lupine). Each combination of these factors leads to a different model. We select the best model using corrected Akaike's information criterion $\mathrm{AIC}_{\mathrm{c}}$ and report Akaike weights (Burnham and Anderson 2002). For nested models, we also report the results of a likelihood-ratio test (Pawitan 2001).

The simplest model of survival is that all individuals experience the same instantaneous daily mortality rate $k$. Then, the probability of each IJ surviving $t$ days is $S(t)=\mathrm{e}^{-k t}$, and the number of IJ surviving in a tube after a period time is a binomial random variable. The mean of this binomial variable is the product of the initial number of IJ in the tube $N_{0}$ and their survival probability; thus $N_{0} \mathrm{e}^{-k t}$.

Heterogeneity in survival across tubes will result in a variance of the number of survivors that is higher than predicted by the binomial. This extra-binomial variability (over-dispersion) is common in count data and can be addressed with a beta binomial model (Dorazio and Royle 2003; McCullagh and Nelder 1989). The beta binomial model arises when data follow a binomial distribution with 
a probability of success that is itself a random variable with a beta distribution (cf. Griffiths 1973). Thus, the beta binomial is a natural choice for modeling between-tube heterogeneity; the number of survivors in a tube follows a binomial random variable with a mean drawn from a beta distribution. The resulting beta binomial distribution is

$$
\begin{gathered}
P(Y=y)=\left(\begin{array}{c}
N_{0} \\
y
\end{array}\right)\left(\frac{B\left(y+\alpha, N_{0}-y+\beta\right)}{B(\alpha, \beta)}\right) \\
y=0,1, \ldots, N_{0}
\end{gathered}
$$

where $y$ is the number of surviving $\mathrm{IJ}, B$ is the beta function, and $\alpha, \beta$ are positive parameters. Using the re-parameterization $\pi=\frac{\alpha}{\alpha+\beta}$ and $\theta=\frac{1}{\alpha+\beta}$, this distribution has mean $N_{0} \pi$ and variance $N_{0} \pi(1-\pi) \frac{1+N_{0} \theta}{1+\theta}$ (Dorazio and Royle 2003). Parameters $\alpha, \beta$, and $\pi$ all depend on time, but to simplify notation we do not explicitly indicate so. Additionally, $\pi$ is the mean of the distribution of survival probabilities (Griffiths 1973). Thus, we set $\pi=S(t)\left(\mathrm{e}^{-k t}\right.$ in the case of no individual heterogeneity).

We model individual heterogeneity in a similar way. We assume that each individual IJ has a mortality rate $k$, which is drawn from a gamma distribution. More specifically, if $T$ is the random variable that gives the lifespan of an individual IJ, then the conditional density function of $T$ given mortality rate $k$ is $f_{T \mid k}(t \mid k)=k \mathrm{e}^{-k t}$, and the density function for $k$ is

$f_{K}(k)=\frac{\alpha^{\alpha}}{\mu^{\alpha} \Gamma(\alpha)} k^{\alpha-1} \mathrm{e}^{-\frac{\alpha}{\mu} k}$

where $\mu$ is the mean and $\frac{\mu^{2}}{\alpha}$ is the variance. Then the density function for IJ lifespan is

$$
\begin{aligned}
f_{T}(t) & =\int_{0}^{\infty} f_{T \mid k}(t \mid k) f_{K}(k) \mathrm{d} k \\
& =\int_{0}^{\infty} k \mathrm{e}^{-k t} \frac{\alpha^{\alpha}}{\mu^{\alpha} \Gamma(\alpha)} k^{\alpha-1} \mathrm{e}^{-\frac{\alpha}{\mu} k} \mathrm{~d} k \\
& =\frac{\alpha\left(\frac{\alpha}{\mu}\right)^{a}}{\left(t+\frac{\alpha}{\mu}\right)^{\alpha+1}} .
\end{aligned}
$$

This distribution is called a Pareto (McNolty et al. 1980) and is mathematically equivalent to the zero term of the negative binomial used in the classic stabilization of Nicholson-Bailey model (Anderson and May 1978; Southwood 1978). Then, the probability that an IJ survives $t$ days is

$$
\begin{aligned}
S(t) & =P(T>t) \\
& =\int_{t}^{\infty} f_{T}(\tau) \mathrm{d} \tau=\left(1+\frac{\mu}{\alpha} t\right)^{-\alpha} .
\end{aligned}
$$

This function is called the survival function $S(t)$, and the hazard function is given by $-\frac{S^{\prime}(t)}{S(t)}$ (Cox and Oakes 1984). In this context, the hazard function represents the mean mortality rate of the remaining individuals.

The nature of heterogeneity determines the structure of the model. We model between-tube heterogeneity by a beta binomial distribution with $\pi=S(t)$; otherwise, we use a binomial distribution. Individual heterogeneity determines how the survival function $S(t)$ for each tube depends on time; heterogeneity yields the Pareto-based model, while homogeneity yields an exponential model. It is important to note that our models do not explicitly model the variability in the distribution of individual survival rates across tubes. Such a model would be intractable; instead, we use the beta-binomial to model heterogeneity in the probability of survival across tubes. However, we test the performance of the model on simulated data that are generated with more realistic assumptions about variability in survival across tubes (see "Simulations"). We use the notation $M_{w b r}$ to describe each model, where the subscripts $w, b$, and $r$ are 0 or 1 to denote the existence or not of within tube heterogeneity, between-tube heterogeneity, and rhizosphere effect, respectively. The eight models are summarized in Table 1. To account for mortality caused by transfer to the tubes, we present versions of these models that include an initial instantaneous mortality event in supporting information $\mathrm{C}$.

We fit each model using maximum likelihood. Formulas for the likelihood functions and deviance residuals are derived using standard techniques and are given in Supporting information $\mathrm{A}$. To fit all the models, we wrote software in the MATLAB computing environment to find the maximum likelihood estimate. To find the optimal set of parameters, we took the best output from a large number of runs of the fminsearch function, which uses the NelderMead simplex algorithm (Lagarias et al. 1998). Each run was initialized with randomly selected parameter values. The number of runs was chosen so that the code produced consistent results; for the models with a larger number of parameters, we had to use as many as 1,000 runs. Because not all our models are nested, we use corrected Akaike information criteria $\left(\mathrm{AIC}_{\mathrm{c}}\right)$ and Akaike weights $w_{i}$ to determine the best-fitting model (Burnham and Anderson 2002). We compare our models that are nested using a likelihood-ratio test (Pawitan 2001). MATLAB code is listed in supporting information B.

\section{Simulations}

We test our statistical analysis on simulated data where the mean of the distribution of individual survival rates varies across the sample units (tubes). In the simulations, the 
Table 1 Summary of models

\begin{tabular}{llllll}
\hline Model & $\begin{array}{l}\text { Within-tube } \\
\text { heterogeneity }\end{array}$ & $\begin{array}{l}\text { Between-tube } \\
\text { heterogeneity }\end{array}$ & $\begin{array}{l}\text { Plant } \\
\text { effect }\end{array}$ & Distribution & Survival $S(t)$ \\
\hline$M_{000}$ & No & No & No & Binomial & $\mathrm{e}^{-k t}$ \\
$M_{001}$ & No & No & Yes & Binomial & $\mathrm{e}^{-k_{r} t}$ \\
$M_{010}$ & No & Yes & No & Beta Binomial & $\mathrm{e}^{-k t}$ \\
$M_{011}$ & No & Yes & Yes & Beta binomial & $\mathrm{e}^{-k_{r} t}$ \\
$M_{100}$ & Yes & No & No & Binomial & $\left(1+\frac{\mu}{\alpha} t\right)^{-\alpha}$ \\
$M_{101}$ & Yes & No & Yes & Binomial & $\left(1+\frac{\mu_{r}}{\alpha_{r}}\right)^{-\alpha_{r}}$ \\
$M_{110}$ & Yes & Yes & No & Beta binomial & $\left(1+\frac{\mu}{\alpha} t\right)^{-\alpha}$ \\
$M_{111}$ & Yes & Yes & Yes & Beta binomial & $\left(1+\frac{\mu_{r}}{\alpha_{r}}\right)^{-\alpha_{r}}$ \\
\hline
\end{tabular}

mean mortality rate $m$ in each tube is drawn from a gamma distribution, with mean $\mu$ and variance $\frac{\mu^{2}}{\phi}$. Then, given $m$, the individual survival rates within a tube is gamma distributed with mean $m$ and variance $\frac{m^{2}}{\alpha}$. Thus, given $m$, an individual's lifespan has a Pareto distribution as before.

All simulated data were generated using MATLAB and our analysis was applied to the simulated data. For all simulations, $\mu$ and $\alpha$ were set to the value found from the real data. We did not address the effect of rhizosphere type in the simulations and assumed that individual and between-tube heterogeneity were both significant. The parameter $\phi$ is analogous to from the beta binomial, except that larger values of $\phi$ correspond to lower levels of between-tube heterogeneity whereas the variance in the number of survivors is a decreasing function of $\theta$. We apply our statistical analysis to the simulated data for varying values of $\phi$ and various simulated sample sizes. In Supporting information D, we compare the results from a simulated non-destructive sampling method to simulated destructively sampled data in the absence of between-tube heterogeneity.

\section{Results}

The data show that nematode survival is heterogeneous among individuals and across tubes and suggest that rhizosphere type may not have a significant effect on survival. Although soil moisture declined at the start of the experiment from 24 to $18 \%$ in 4 days, moisture did not vary significantly over the remainder of the experiment. The model that incorporated both within and between sample heterogeneity but no rhizosphere type effect $M_{110}$ had the lowest $\mathrm{AIC}_{\mathrm{c}}$ with Akaike weight $w=0.567$ and the next best fitting model $M_{111}$ had a $\Delta_{i} \mathrm{AIC}_{\mathrm{c}}=0.3(w=0.463)$ (Table 2). All other models are much further than $10 \mathrm{AIC}_{\mathrm{c}}$ units away from the best-fitting model and Akaike weights $<10^{-6}$, so it is clear they provide a significantly poorer description of the data (Burnham and Anderson 2002). Model $M_{110}$ is nested within model $M_{111}$, so we used a likelihood-ratio test that suggested that $M_{110}$ is the best model $\left(\chi^{2}=3.90, d f=2, P=0.14\right)$. However, Kendall (1998) found the criterion $P>0.19$ in a likelihood ratio test produced a Type I error rate of 0.05 in simulations of a similar model. The fitted parameters for model $M_{110}$ are $\mu=2.89, \alpha=0.774$, and $\theta=0.0520$, and the parameters for model $M_{111}$ are $\mu_{l}=4.32, \alpha_{l}=0.669, \mu_{g}=2.08$, $\alpha_{g}=0.896$, and $\theta=0.0520$. Figure 1 shows histograms for the initial distributions of individual heterogeneity for models $M_{110}$ and $M_{111}$. The parameters for the best fitting exponential model $M_{010}, k_{a}=0.105$, and $\theta=0.850$.

The data do not have an exponentially decaying mean (Fig. 2) and clearly deviate from a straight line on a loglinear plot. However, notice that we plot the log of the number of survivors plus one in order to show the zeros. The departure from the exponential model is evident in deviance residual plots (Fig. 2), which show a clear trend.

Model $M_{110}$ yields more information about heterogeneity in the population. As time progresses, individuals with higher mortality rates are more likely to die, this distribution shifts, and the population mean mortality rate decreases over time (Fig. 3). This is also apparent in Fig. 2, because the exponential model $M_{010}$, which assumes constant population mean mortality, initially overestimates the number of survivors and then underestimates survivors.

Table 2 Comparison of models with corrected Akaike's information criteria $\mathrm{AIC}_{\mathrm{c}}$ and Akaike weights $w_{i}$ (Burnham and Anderson 2002)

\begin{tabular}{lccl}
\hline Model & $\mathrm{AIC}_{\mathrm{c}}$ & $\Delta_{i} \mathrm{AIC}_{\mathrm{c}}$ & $w_{i}$ \\
\hline$M_{110}$ & $1,285.4$ & 0 & 0.537 \\
$M_{111}$ & $1,285.6$ & 0.3 & 0.463 \\
$M_{011}$ & $1,851.9$ & 566.5 & $<10^{-6}$ \\
$M_{010}$ & $1,852.4$ & 567.1 & $<10^{-6}$ \\
$M_{101}$ & $1,892.5$ & 607.1 & $<10^{-6}$ \\
$M_{100}$ & $1,901.9$ & 616.5 & $<10^{-6}$ \\
$M_{001}$ & $6,706.6$ & $5,421.3$ & $<10^{-6}$ \\
$M_{000}$ & $6,719.0$ & $5,433.6$ & $<10^{-6}$ \\
\hline
\end{tabular}



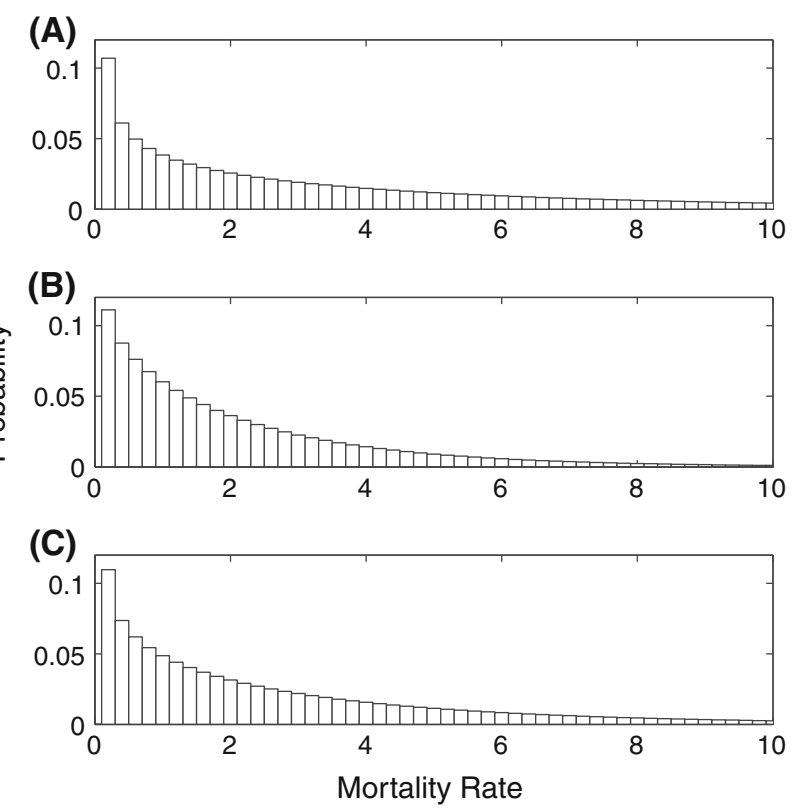

Fig. 1 Histograms of initial distributions of individual mortality rate from models $M_{111}$ and $M_{110}$. We show histograms because the actual probability density functions are difficult to interpret. $\mathbf{a}, \mathbf{b}$ Nematodes under lupines and grassland, respectively, estimated by $M_{111}$, and c for model $M_{110}$
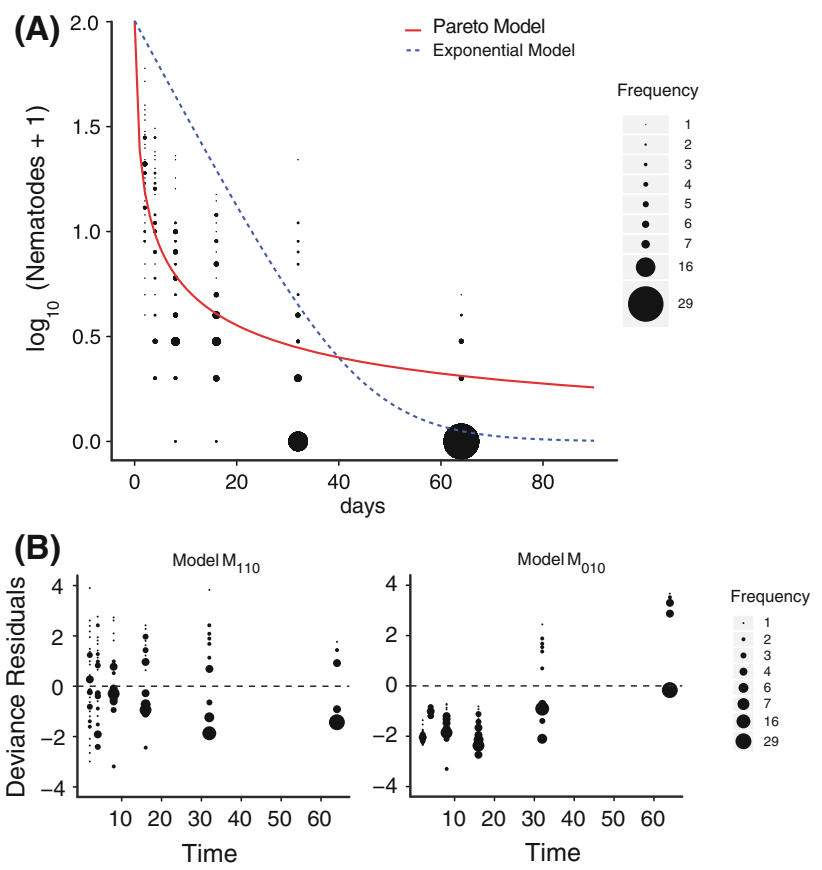

Fig. 2 A comparison of the means of models $M_{110}$ (solid line) and $M_{010}$ (dashed line). Data are shown with circles in (a). b A comparison of the deviance residuals of these models

Figure 4 illustrates the effect of individual heterogeneity on a cohort of 100,000 nematodes extrapolated over a 160-day period based on our model results. A large

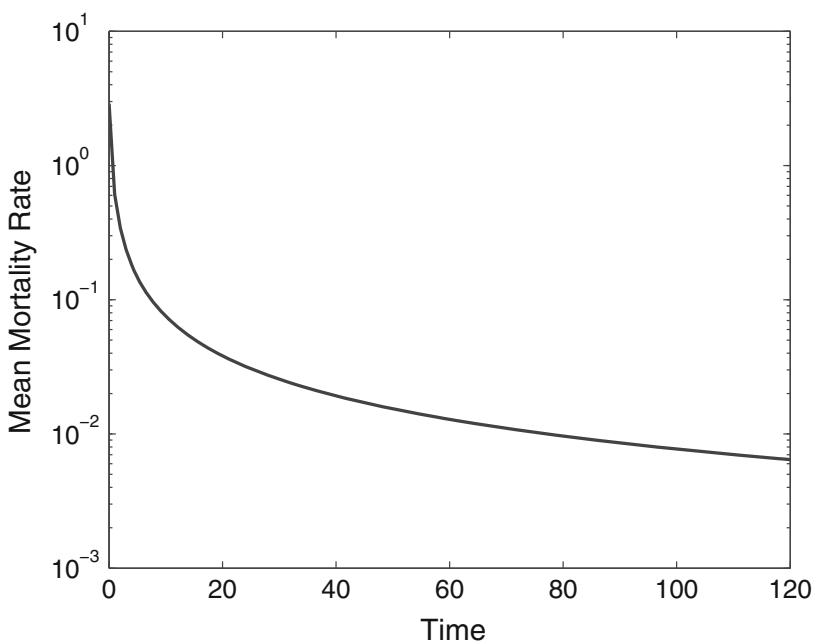

Fig. 3 Mean population mortality rate (i.e. hazard rate) through time derived from model $M_{110}$

naturally occurring Hepialus larva can produce upwards of 100,000 IJ (Strong 2002), and these IJ typically have to survive 5 months before encountering similarly sized hosts. Individual heterogeneity leads to the prediction that a population of individuals will persist over the time period. The assumption of homogeneity predicts population extirpation as the almost certain outcome.

The simulations show that the fitted value of $\theta(\hat{\theta})$ is a decreasing function of $\phi$ as expected (see Fig. 4). With $\mu$ and $\alpha$ set equal to the values generated by the real data, $\phi=$ 1.88 produces the value of $\theta$ that was observed. Our analysis estimates the mean mortality rate $\mu$ with little or no bias, but for high levels of between-tube heterogeneity (low values of $\theta$ ), there is a significant bias in the estimate

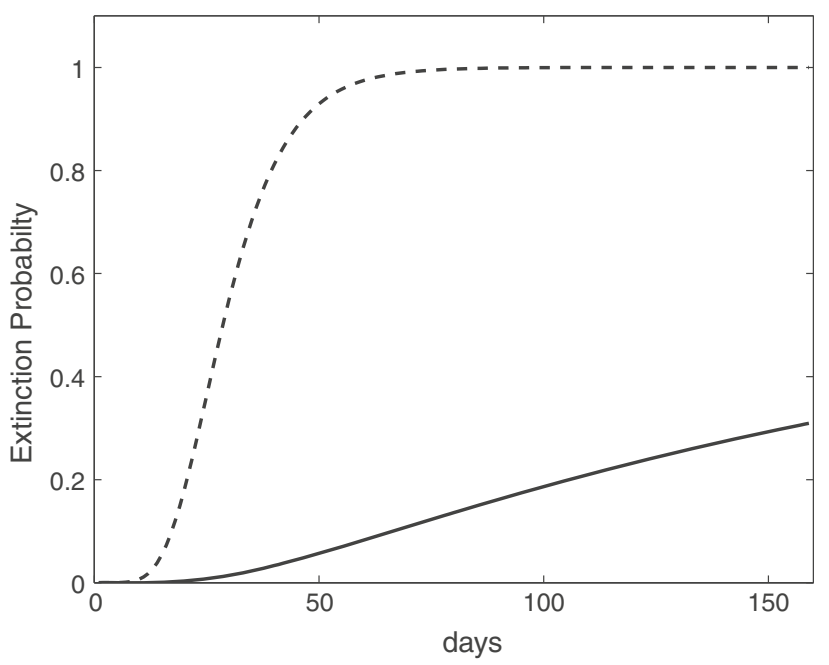

Fig. 4 Probability of extinction for a cohort of nematodes with an initial nematode population $N_{0}=100,000$. Other population parameters are estimated from data. Results for model $M_{110}$ are marked with a solid line and results for model $M_{011}$ are marked with a dashed line 
of $\alpha$, the individual heterogeneity (see Fig. 4). This bias persists as the sample size is increased. The simulation that corresponded to the data $(\phi=1.88, \mu=2.89, \alpha=0.774$, and sample size 40 individuals per tube) had standard deviations of $0.012,0.51$, and 0.043 for $\hat{\theta}, \hat{\mu}$, and $\hat{\alpha}$, respectively (Fig. 5).

\section{Discussion}

Many types of natural enemies such as pathogens, parasitoids, and entomopathogenic nematodes exist in nature in non-feeding free-living stages. Often, these propagules must endure extended periods of unfavorable abiotic or biotic limitations before finding a host. Mechanisms that allow such parasites to persist over the long-term have long been debated (Fenton and Hudson 2002). H. marelatus
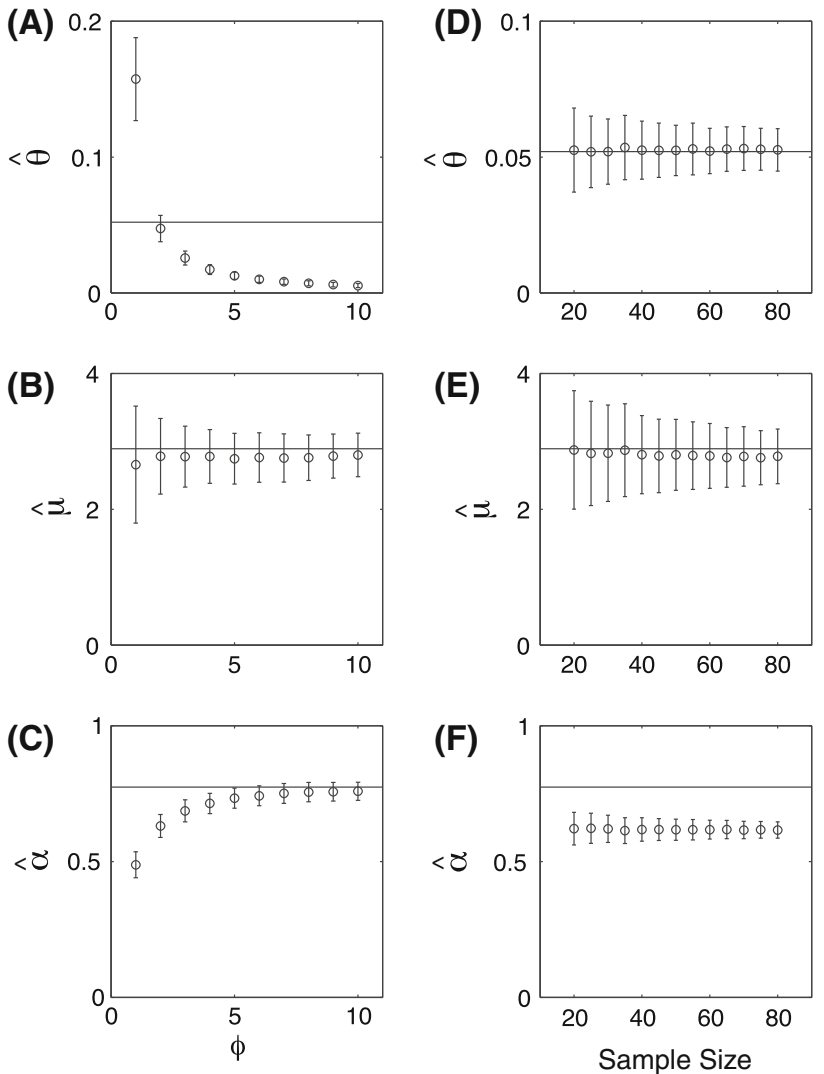

Fig. 5 Results of simulations. a-c The effect of varying the parameter $\phi$ which describes the between-tube variability in mortality rate on fitted parameters $\hat{\theta}, \hat{\mu}$, and $\hat{\alpha}$, respectively. $\mathbf{d}-\mathbf{f}$ The effect of varying the sample size (tubes per sample date) on fitted parameters $\hat{\theta}, \hat{\mu}$, and $\hat{\alpha}$, respectively when $\phi$. Circles represent mean 1,000 simulations and error bars $\pm \mathrm{SD}$. The solid horizontal lines represent the values of the parameters observed in the real data. Parameters $\mu=2.89, \alpha=0.774$ in all panels, the sample size is 40 in $(\mathbf{a}-\mathbf{c})$ and $\phi=1.88$ in $(\mathbf{d}-\mathbf{f})$ individuals produced at the end of spring must endure hot, dry summer conditions in the California coastal prairie until increased seasonal soil moisture and host availability make reproduction feasible. Thus, differing mortality rates among populations and habitats will determine future reproduction and long-term persistence. Multiple long-term studies on $H$. marelatus have yielded average daily mortality rates that are much lower than average daily mortality rates measured over short time periods (Dugaw et al. 2004; Preisser et al. 2006; Ram et al. 2008b). Our results provide an explanation for this pattern: the population average daily mortality rate decreases over time as the weak individuals die off. Previous efforts assumed ecological equivalency among individuals and identical conditions among replicate populations to maintain model tractability; here, we explicitly compare both forms of heterogeneity and demonstrate improved model fit. Heterogeneous mortality is particularly important for $\mathrm{H}$. marelatus, since the ability to survive the hot and dry summers is a key to population persistence (Dugaw et al. 2004).

The precise source of survivorship heterogeneity, however, remains unclear. Two sources of heterogeneity may be driving the system. One source comes from biotic heterogeneity among individuals. A second source is micro-site variation in soil moisture even within a single rhizosphere microhabitat. Soil aggregates which retain moisture may reduce stress and lower mortality (Grant and Villani 2003). In this study, soil moisture remained $>12 \%$, which is considered to be highly conducive for IJ survival and host finding (Grant and Villani 2003). To estimate the relative effects of these factors, our statistical analysis could be applied to a survival experiment comparing heterogeneous media (natural soil) with homogeneous media (sand). However, it is likely that biotic and abiotic heterogeneity interact to regulate mortality rate of EPN in the field.

Unlike previous studies (Preisser et al. 2005; Ram et al. 2008a), we did not find strong evidence for increased survival under lupines. The difference in $\mathrm{AIC}_{\mathrm{c}}$ between models that did and did not include the effect of plant type was very small (0.3), but the $P$ value from the likelihood ratio test was 0.14 . However, many of the parameters estimates for each habitat type lie further than 2 standard deviations away from the parameters for the combined model $\left(M_{110}\right)$. The impact of these parameter values on the overall initial distribution of mortality rates is minimal (see Fig. 1). As previously mentioned, the work of Kendall (1998) suggests that the likelihood ratio test $P$ value may not accurately reflect the true Type I error rate. This issue can be addressed directly with additional simulation work, which we did not pursue because our focus was on heterogeneity.

Since an individual's frailty cannot be measured directly, this indirect analysis of heterogeneity is consistent 
with other possible explanations for the patterns in the data. For example, it is possible that all individuals are essentially identical and that they become more robust as they age. This is unlikely in our system because the IJ nematodes do not feed. Another possible explanation for the data is density-dependent mortality, i.e. as individuals die the remaining individuals have higher survival due to lower density. This is also unlikely in our system because the non-feeding IJ do not compete for resources, and they have no known natural enemies that regulate populations in a density-dependent manner (Jaffee and Strong 2005); also see Appendix A in Ram et al. 2008b).

In this study, we demonstrate the use of a technique to test for heterogeneity in survival of organisms that are difficult to observe without destructive sampling or are impractical to sample more than once. We acknowledge the caveat that our model may not adequately describe dynamics of highly mobile organisms that are capable of complex behavioral decisions. Several previous statistical analyses of heterogeneous survival have relied on data on the lifespans of large numbers of humans (Yashin et al. 1985), med flies (Vaupel and Carey 1993), and scrubjays (Fox et al. 2006). Such large datasets required to fit these models are not readily available for most natural populations (Fox et al. 2006, for example, relied on a $>35$-year study comprised of monthly censuses). Additionally, most of these models assume independence among samples. Such an assumption is too strong for most practical experimental designs where individuals are grouped by sampling unit or other hierarchical structure (Stauffer 2008). Our statistical model accommodates hierarchical heterogeneity and destructive sampling of populations. Our simulations showed that our simplifying assumptions about the nature of heterogeneity introduced a minimal bias in the mean individual mortality rate and a $19 \%$ bias in the estimate of the $\alpha$ (a measure of individual heterogeneity). Thus, our model is readily applicable to populations for which repeated sampling is impractical or impossible, e.g., subterranean organisms, internal macroparasites, seed-sets, and non-reproducing infectious propagules of various microparasites. Extrapolating our results to larger populations yielded the interesting result that heterogeneous survival can have a significant effect on the emergent number of survivors in large populations (Fig. 4). Additional heterogeneity arising from differences in soil properties, particularly with respect to moisture retention, can also be included as additional interaction effects in the model similar to the rhizospheres terms. It is typically thought that such variability only impacts small populations (Connors and White 1999), which is certainly true for populations with overlapping generations. However, individual heterogeneity in survival will significantly effect seasonal populations that must endure long periods between reproductive events even for large populations.

Our statistical model of heterogeneous survival is perhaps the simplest that allows for a continuous range of mortality rates, and is readily implemented in mathematical models to provide more insight into survivorship. Because our model assumes an individual has constant mortality rate over its lifespan, it is more readily incorporated into a mathematical model than a model with age-dependent mortality. This simplifying assumption renders our analysis inappropriate for studying senescence, which was the focus of previous works (e.g., Vaupel and Carey 1993; Fox et al. 2006), but a mathematical model with such an assumption is likely to yield valuable results in an ecological context.

Acknowledgments We thank R. Van Kirk, Evan Preisser, Daniel Gruner and Donald Strong for comments on earlier drafts of the manuscript. Discussions with Marc Mangel and the comments of anonymous reviewers greatly improved the quality of the manuscript. K. Ram was supported by a grant from the Bodega Marine Lab and by NSF DEB-0620475. This publication is a contribution of the Bodega Marine Laboratory, University of California at Davis.

Open Access This article is distributed under the terms of the Creative Commons Attribution Noncommercial License which permits any noncommercial use, distribution, and reproduction in any medium, provided the original author(s) and source are credited.

\section{References}

Anderson RM, May RM (1978) Regulation and stability of hostparasite population interactions: I. Regulatory processes. J Anim Ecol 47:219-247

Anderson DR et al (2001) Field trials of line transect methods applied to estimation of desert tortoise abundance. J Wildl Manage 65:583-597

Bolnick DI et al (2003) The ecology of individuals: incidence and implications of individual specialization. Am Nat 161:1-28

Burnham KP, Anderson DR (2002) Model selection and multi-model inference: a practical information-theoretic approach, 2nd edn. Springer, New York

Campbell JF, Koppenhofer AM, Kaya HK, Chinnasri B (1999) Are there temporarily non-infectious dauer stages in entomopathogenic nematode populations: a test of the phased infectivity hypothesis. Parasitology 118:499-508

Connors MM, White GC (1999) Effects of individual heterogeneity in estimating the persistence of small populations. Nat Resour Model 12:109-127

Cox DR, Oakes D (1984) Analysis of survival data (monographs on statistics and applied probability). Chapman \& Hall, London

Czáran T (1998) Spatiotemporal models of population and community dynamics. Chapman \& Hall, London

Darwin C (1859) The origin of species. John Murray, London

Dorazio RM, Royle JA (2003) Mixture models for estimating the size of a closed population when capture rates vary among individuals. Biometrics 59:351-364

Duchateau L, Janssen P (2005) Understanding heterogeneity in generalized mixed and frailty models. Am Stat 59:143-146 
Dugaw CJ, Hastings A, Preisser EL, Strong DR (2004) Seasonally limited host supply generates microparasite population cycles. Bull Math Biol 66:583-594

Elliot A (1954) Relationship of aging, food reserves and infectivity of larvae of Axaridia galli. Exp Parasitol 3:307-320

Fenton A, Hudson PJ (2002) Optimal infection strategies: should macroparasites hedge their bets? Oikos 96:92-101

Fox GA (2005) Extinction risk of heterogeneous populations. Ecology 86:1191-1198

Fox GA, Kendall BE (2002) Demographic stochasticity and the variance reduction effect. Ecology 83:1928-1934

Fox GA, Kendall BE, Fitzpatrick JW, Woolfenden GE (2006) Consequences of heterogeneity in survival probability in a population of Florida scrub-jays. J Anim Ecol 75:921-927

Grant JA, Villani MG (2003) Soil moisture effects on entomopathogenic nematodes. Environ Entomol 32:80-87

Grear JS, Schmitz OJ (2005) Effects of grouping behavior and predators on the spatial distribution of a forest floor arthropod. Ecology 86:960-971

Griffiths DA (1973) Maximum likelihood estimation for the betabinomial distribution and an application to the household distribution of the total number of cases of a disease. Biometrics 29:637-648

Jaffee BA, Strong DR (2005) Strong bottom-up and weak top-down effects in soil: nematode-parasitized insects and nematodetrapping fungi. Soil Biol Biochem 37:1011-1021

Kendall B (1998) Estimating the magnitude of environmental stochasticity in survivorship data. Ecol Appl 8:184-193

Kendall BE, Fox GA (2003) Unstructured individual variation and demographic stochasticity. Conserv Biol 17:1170-1172

Kowald A, Kirkwood TBL (1993) Explaining fruit-fly longevity. Science 260:1664-1665

Kung S, Gaugler R (1990) Soil type and entomopathogenic nematode persistence. J Invertebr Pathol 55:401-406

Kung S, Gaugler R (1991) Effects of soil temperature, moisture, and relative humidity on entomopathogenic nematode persistence. J Invertebr Pathol 57:242-249

Lagarias JC, Reeds JA, Wright MH, Wright PE (1998) Convergence properties of the Nelder-Mead simplex method in low dimensions. SIAM J Optim 9:112-147

Lewis EE, Campbell J, Griffin C, Kaya H, Peters A (2006) Behavioral ecology of entomopathogenic nematodes. Biol Control 38:66-79

Lomnicki A (1980) Regulation of population-density due to individual-differences and patchy environment. Oikos 35:185-193

McCullagh P, Nelder JA (1989) Generalized linear models, 2nd edn. Chapman \& Hall, London

McNolty F, Doyle J, Hansen E (1980) Properties of mixed exponential failure process. Technometrics 22:555-565

Pacala SW (1987) Neighborhood models of plant-population dynamics. 3. Models with spatial heterogeneity in the physicalenvironment. Theor Popul Biol 31:359-392

Pawitan Y (2001) In all likelihood: statistical modelling and inference using likelihood. Oxford University Press, New York

Pfister CA, Stevens FR (2003) Individual variation and environmental stochasticity: implications for matrix model predictions. Ecology $84: 496-510$
Pickett STA, Cadenasso ML, Jones CG (2000) Generation of heterogeneity: creation, maintenance and transformation. In: Hutchings MJ, John EA, Stewart AJA (eds) The ecological consequences of environmental heterogeneity. British Ecological Society, Oxford

Preisser EL (2003) Field evidence for a rapidly cascading underground food web. Ecology 84:869-874

Preisser EL, Dugaw CJ, Dennis B, Strong DR (2005) Long-term survival of the entomopathogenic nematode Heterorhabditis marelatus. Environ Entomol 34:1501-1506

Preisser EL, Dugaw CJ, Dennis B, Strong DR (2006) Plant facilitation of a belowground predator. Ecology 87:1116-1123

Qiu L, Bedding R (2000) Energy metabolism and its relation to survival and infectivity of infective juveniles of Steinernema carpocapsae under aerobic conditions. In: Nematology, vol 2. VSP, Netherlands, pp 551-559

Ram K, Gruner DS, Mclaughlin JP, Preisser EL, Strong DR (2008a) Long-term dynamics of a subterranean trophic cascade in space and time. J Nematol 40:85-92

Ram K, Preisser EL, Gruner DS, Strong D (2008b) Metapopulation dynamics override local limits on long-term parasite persistence. Ecology 89:3290-3297

Robert A, Sarrazin FO, Couvet D (2003) Variation among individuals, demographic stochasticity, and extinction: response to Kendall and Fox. Conserv Biol 17:1166-1169

Schoener TW (1986) Resource partitioning. In: Kilkkawa J, Anderson DJ (eds) Community ecology pattern and process. Blackwell, Boston

Service PM, Michieli CA, McGill K (1998) Experimental evolution of senescence: an analysis using a "heterogeneity" mortality model. Evolution 52:1844-1850

Smits PH (1996) Post-application persistence of entomopathogenic nematodes. Biocontrol Sci Technol 6:379-388

Southwood TRE (1978) Ecological methods, with particular reference to the study of insect populations. Chapman \& Hall, London

Stauffer HB (2008) Contemporary Bayesian and frequentist statistical research methods for natural resource scientists. Wiley, London

Strong D (1999) Predator control in terrestrial ecosystems: the underground food chain of bush lupine. In: Olff $\mathrm{H}$, Brown VK, Drent RH (eds) Herbivores, between plants and predators. Blackwell, Oxford, pp 577-602

Strong DR (2002) Populations of entomopathogenic nematodes in food webs. In: Gaugler R (ed) Entomopathogenic nematology. CABI, NY, pp 225-240

Strong DR et al (1996) Entomopathogenic nematodes: natural enemies of root-feeding caterpillars on bush lupine. Oecologia 108:167-173

Vaupel JW, Carey JR (1993) Compositional interpretations of medfly mortality. Science 260:1666-1667

Vindenes Y, Engen S, Saether BE (2008) Individual heterogeneity in vital parameters and demographic stochasticity. Am Nat 171: 455-467

Yashin AI, Manton KG, Vaupel JW (1985) Mortality and aging in a heterogeneous population-a stochastic-process model with observed and unobserved variables. Theor Popul Biol 27:154175 